\title{
SOME NEW SUBSPACES OF AN FK-SPACE AND DEFERRED CESÀRO CONULLITY
}

\author{
ŞEYDA SEZGEK AND İLHAN DAĞADUR
}

\begin{abstract}
In this paper, we construct new important subspaces $D_{p}^{q} S, D_{p}^{q} W, D_{p}^{q} F$ and $D_{p}^{q} B$ for a locally convex FK-space $X$ containing $\phi$, the space of finite sequences. Then, we show that there is a relation among these subspaces. Also, we study deferred Cesàro conullity of one FK-space with respect to another, and we give some important results. Finally, we examine the deferred Cesàro conullity of the absolute summability domain $l_{A}$, and show that if $l_{A}$ is deferred Cesàro conull, then $A$ cannot be $l$-replaceable.
\end{abstract}

\section{INTRODUCTION}

Let $w$ denote the space of all complex valued sequences. It can be topologized with the seminorms $r_{n}(x)=\left|x_{n}\right|, n=1,2, \ldots$, and any vector subspace $X$ of $w$ is a sequence space. A sequence space $X$ with a vector space topology $\tau$ is a $\mathrm{K}$-space provided that the inclusion map $i:(X, \tau) \rightarrow w, i(x)=x$, is continuous. If, in addition, $\tau$ is complete, matrizable, locally convex, then $(X, \tau)$ is called an FK-space. So, an FK-space is a complete, metrizable locally convex topological vector space of sequences for which the coordinate functionals are continuous. An FK-space whose topology is normable is called a BK-space. The basic properties of an FK-space may be found in (see $[2,11,13]$ ).

By $c, c_{0}, l_{\infty}$, we denote the spaces of convergent sequences, null sequences and bounded sequences, respectively. These are FK-spaces under $\|x\|=\sup _{n}\left|x_{n}\right|$. By $c s, l$, we denote the spaces of all summable sequences, absolute summable sequences, respectively.

Throughout this paper, $e$ denotes the sequences of ones; $\delta^{j}(j=1,2, \ldots)$ the sequence with the one in the $j$-th position; $\phi$ the linear span of $\delta^{j}$ 's. The linear span of $\phi$ and $e$ is denoted by $\phi_{1}$. The topological dual of $X$ is denoted by $X^{\prime}$. A sequence $x$ in a locally convex sequence space $X$ is said to be the property AK if $x^{(n)} \rightarrow x$ in $X$, where $x^{(n)}=\sum_{k=1}^{n} x_{k} \delta^{k}$. The space $X$ is said to have AD if $\phi$ is dense in $X$.

We recall (see $[2,11]$ ) that the $f, \beta$-duals of a subset $X$ of $w$ are

$$
X^{f}=\left\{\left\{f\left(\delta^{k}\right)\right\}: f \in X^{\prime}\right\},
$$

$M S C$ (2020): primary 46A45, 40A05; secondary 40C05, 40D05.

Keywords: deferred Cesàro mean, deferred Cesàro conull, FK-space, AK-space, $\sigma_{p}^{q}[K]$-space, $\sigma_{p}^{q}[B]$-space. 


$$
X^{\beta}=\left\{x \in w: \sum_{n=1}^{\infty} x_{k} y_{k} \text { is convergent for all } y \in X\right\} .
$$

In 1932, Agnew [1] defined the deferred Cesàro mean $D_{p, q}$ of the sequences $x$ by

$$
\left(D_{p, q} x\right)_{n}=\frac{1}{q(n)-p(n)} \sum_{k=p(n)+1}^{q(n)} x_{k},
$$

where $\{p(n)\}$ and $\{q(n)\}$ are sequences of nonnegative integers satisfying the conditions $p(n)<q(n)$ and $\lim _{n \rightarrow \infty} q(n)=\infty$. We note here that $D_{p, q}$ is clearly regular for any choice of $\{p(n)\}$ and $\{q(n)\}$. We define some new sequence spaces by using a deferred Cesàro mean.

The sequence spaces

$$
\begin{aligned}
{\left[\sigma_{0}\right]_{p}^{q}: } & =\left\{x \in w: \lim _{n} \frac{1}{q(n)-p(n)} \sum_{k=p(n)+1}^{q(n)} x_{k}=0\right\}, \\
{\left[\sigma_{c}\right]_{p}^{q}: } & =\left\{x \in w: \lim _{n} \frac{1}{q(n)-p(n)} \sum_{k=p(n)+1}^{q(n)} x_{k} \text { exists }\right\}, \\
{\left[\sigma_{\infty}\right]_{p}^{q}: } & =\left\{x \in w: \sup _{n} \frac{1}{q(n)-p(n)}\left|\sum_{k=p(n)+1}^{q(n)} x_{k}\right|<\infty\right\}, \\
\sigma_{p}^{q}[s]: & =\left\{x \in w: \lim _{n} \frac{1}{q(n)-p(n)} \sum_{k=p(n)+1}^{q(n)} \sum_{j=1}^{k} x_{j} \text { exists }\right\}
\end{aligned}
$$

and

$$
\sigma_{p}^{q}[b]:=\left\{x \in w: \sup _{n}\left|\frac{1}{q(n)-p(n)} \sum_{k=p(n)+1}^{q(n)} \sum_{j=1}^{k} x_{j}\right|<\infty\right\}
$$

are BK-spaces with the norms

$$
\|x\|_{\left[\sigma_{0}\right]_{p}^{q}}=\sup _{n}\left|\frac{1}{q(n)-p(n)} \sum_{k=p(n)+1}^{q(n)} x_{k}\right|
$$

and

$$
\|x\|_{\sigma_{p}^{q}[s]}=\sup _{n}\left|\frac{1}{q(n)-p(n)} \sum_{k=p(n)+1}^{q(n)} \sum_{j=1}^{k} x_{j}\right| .
$$

This assertion can be proved along the same lines as in (see $[3,4,6,7])$, so we omit the details.

A sequence $x$ in a locally convex sequence space $X$ is said to be the property $\sigma_{p}^{q}[K]$ if

$$
\frac{1}{q(n)-p(n)} \sum_{k=p(n)+1}^{q(n)} x^{(k)} \rightarrow x \quad \text { in } X .
$$


A sequence $x$ in a locally convex sequence space $X$ is said to be the property $\sigma_{p}^{q}[B]$ if $\forall x \in X$

$$
\left\{\frac{1}{q(n)-p(n)} \sum_{k=p(n)+1}^{q(n)} x^{(k)}\right\} \text { is bounded in } X
$$

Now, we define new $d-, d[b]$-type duals of a sequence space $X$ containing $\phi$.

$$
\begin{aligned}
X^{d} & =\left\{x \in w: \lim _{n \rightarrow \infty} \frac{1}{q(n)-p(n)} \sum_{k=p(n)+1}^{q(n)} \sum_{j=1}^{k} x_{j} y_{j} \text { exists for all } y \in X\right\} \\
= & \left\{x \in w: x . y \in \sigma_{p}^{q}[s] \text { for all } y \in X\right\} \\
X^{d[b]}= & \left\{x \in w: \sup _{n} \frac{1}{q(n)-p(n)}\left|\sum_{k=p(n)+1}^{q(n)} \sum_{j=1}^{k} x_{j} y_{j}\right|<\infty, y \in X\right\} \\
= & \left\{x \in w: x \cdot y \in \sigma_{p}^{q}[b] \text { for all } y \in X\right\}
\end{aligned}
$$

respectively, where $x \cdot y=\left(x_{n} y_{n}\right)$.

Let $X, Y$ be sets of sequences. Then, for $\nu=f, \beta, b, d[b]$,

(i) $X \subset X^{\nu \nu}$,

(ii) $X^{\nu \nu \nu}=X^{\nu}$,

(iii) If $X \subset Y$, then $Y^{\nu} \subset X^{\nu}$ holds.

Theorem 1.1. Let $X$ be an FK-space $\supset \phi$, and

$$
\lim _{n \rightarrow \infty} \frac{q(n)-i+1}{q(n)-p(n)}=1 \quad(i \leq q(n)) .
$$

Then,

(i) $X^{\beta} \subset X^{d} \subset X^{d[b]} \subset X^{f}$,

(ii) if $X$ is a $\sigma_{p}^{q}[K]$-space, then $X^{f}=X^{d}$

and

(iii) if $X$ is an AD-space, then $X^{d[b]}=X^{d}$.

Proof. (ii) Suppose that $u \in X^{d}$ and

$$
f(x)=\lim _{n \rightarrow \infty} \frac{1}{q(n)-p(n)} \sum_{k=p(n)+1}^{q(n)} \sum_{j=1}^{k} x_{j} u_{j}
$$

for $x \in X$. Then, $f \in X^{\prime}$ by the Banach-Steinhaus theorem. Now, we get

$$
f\left(\delta^{i}\right)=\lim _{n \rightarrow \infty} \frac{1}{q(n)-p(n)} \sum_{k=p(n)+1}^{q(n)} \sum_{j=1}^{k} u_{j} \delta^{i}=\lim _{n \rightarrow \infty} \frac{q_{n}-i+1}{q(n)-p(n)} u_{i}=u_{i},
$$

so $u \in X^{f}$. Thus, $X^{d} \subset X^{f}$. 
Let $u \in X^{f}$. Since $X$ is a $\sigma_{p}^{q}[K]$-space, we have

$$
\begin{aligned}
f(x) & =\lim _{n \rightarrow \infty} \frac{1}{q(n)-p(n)} \sum_{k=p(n)+1}^{q(n)} \sum_{j=1}^{k} x_{j} f\left(\delta^{j}\right) \\
& =\lim _{n \rightarrow \infty} \frac{1}{q(n)-p(n)} \sum_{k=p(n)+1}^{q(n)} \sum_{j=1}^{k} x_{j} u_{j}
\end{aligned}
$$

for all $x \in X$. Then, $u \in X^{d}$. That means $X^{f} \subset X^{d}$. This proves (ii).

(iii) Let $u \in X^{d[b]}$. Assume that

$$
f_{n}(x)=\frac{1}{q(n)-p(n)} \sum_{k=p(n)+1}^{q(n)} \sum_{j=1}^{k} u_{j} x_{j}
$$

for all $x \in X$. Then, $\left\{f_{n}\right\}$ is pointwise bounded and hence equicontinuous by [11]. Since

$$
\lim _{n \rightarrow \infty} f_{n}\left(\delta^{i}\right)=u_{i}, \quad i<q(n),
$$

$\phi \subset\left\{x: \lim _{n} f_{n}(x)\right.$ exists $\}$ is a closed subspace of $X$ by the Convergence lemma (see [11]). Also, since $X$ is an AD-space, $X=\left\{x: \lim _{n} f_{n}(x)\right.$ exists $\}=\bar{\phi}$, hence $\lim _{n} f_{n}(x)$ exists for all $x \in X$. So, $u \in X^{d}$. The opposite inclusion is trivial.

(i) By the hypothesis, $\bar{\phi} \subset X$. Since $\bar{\phi}$ is an AD-space, by (ii), (iii) and [11, (7.2.4)], we get

$$
X^{d[b]} \subset(\bar{\phi})^{d[b]}=(\bar{\phi})^{d} \subset(\bar{\phi})^{f}=X^{f} .
$$

\section{MAin RESUlts}

We shall define some new subspaces of a locally convex FK-space $X$ containing $\phi$, the space of finite sequences, which are of importance for each one in the topological sequence spaces theory.

Definition 2.1. Let $X$ be an FK-space $\supset \phi$. Then,

$$
\begin{aligned}
& D_{p}^{q} W:=D_{p}^{q} W(X)=\left\{x \in X: \frac{1}{q(n)-p(n)} \sum_{k=p(n)+1}^{q(n)} x^{(k)} \rightarrow x \text { (weakly) in } X\right\} \\
&=\left\{x \in X: f(x)=\lim _{n} \frac{1}{q(n)-p(n)} \sum_{k=p(n)+1}^{q(n)} \sum_{j=1}^{k} x_{j} f\left(\delta^{j}\right) \text { for all } f \in X\right\}, \\
& D_{p}^{q} S:=D_{p}^{q} S(X)=\left\{x \in X: \frac{1}{q(n)-p(n)} \sum_{k=p(n)+1}^{q(n)} x^{(k)} \rightarrow x\right\} \\
&=\left\{x \in X: x \text { has } \sigma_{p}^{q}[K] \operatorname{in} X\right\}
\end{aligned}
$$




$$
=\left\{x \in X: x=\lim _{n} \frac{1}{q(n)-p(n)} \sum_{k=p(n)+1}^{q(n)} \sum_{j=1}^{k} x_{j} \delta^{j}\right\} .
$$

Thus, $X$ is a $\sigma_{p}^{q}[K]$-space if and only if $D_{p}^{q} S=X$.

$$
\begin{aligned}
D_{p}^{q} F^{+} & :=D_{p}^{q} F^{+}(X) \\
& =\left\{x \in w: \lim _{n}\left\{\frac{1}{q(n)-p(n)} \sum_{k=p(n)+1}^{q(n)} x^{(k)}\right\} \text { is weakly Cauchy in } X\right\} \\
& =\left\{x \in w: \lim _{n} \frac{1}{q(n)-p(n)} \sum_{k=p(n)+1}^{q(n)} \sum_{j=1}^{k} x_{j} f\left(\delta^{j}\right) \text { exists for all } f \in X^{\prime}\right\} \\
& =\left\{x \in w:\left\{x_{n} f\left(\delta^{n}\right)\right\} \in \sigma_{p}^{q}[s] \text { for all } f \in X^{\prime}\right\}=\left(X^{f}\right)^{d} . \\
D_{p}^{q} B^{+}:= & D_{p}^{q} B^{+}(X)=\left\{x \in w:\left\{\frac{1}{q(n)-p(n)} \sum_{k=p(n)+1}^{q(n)} x^{(k)}\right\} \text { is bounded in } X\right\} \\
& =\left\{x \in w:\left(x_{n} f\left(\delta^{n}\right)\right) \in \sigma_{p}^{q}[b] \text { for all } f \in X^{\prime}\right\}
\end{aligned}
$$

also $D_{p}^{q} F=D_{p}^{q} F^{+} \cap X$ and $D_{p}^{q} B=D_{p}^{q} B^{+} \cap X$.

We now study some inclusions which are analogous to those given in [11, Chapter 10]. Also, we prove some theorems related to the $f$-, $d$ - and $d[b]$-duality of a sequence space $X$.

Theorem 2.2. Let $X$ be an FK-space $\supset \phi$. Then,

$$
\phi \subset D_{p}^{q} S \subset D_{p}^{q} W \subset D_{p}^{q} F \subset D_{p}^{q} B \subset X \text { and } \phi \subset D_{p}^{q} S \subset D_{p}^{q} W \subset \bar{\phi} .
$$

Proof. The only non-trivial part is $D_{p}^{q} W \subset \bar{\phi}$. Let $f \in X^{\prime}$ and $f=0$ on $\phi$. The definition of $D_{p}^{q} W$ shows that $f=0$ on $D_{p}^{q} W$. Hence, the Hahn-Banach theorem gives the result.

Theorem 2.3. The subspaces $E=D_{p}^{q} S, D_{p}^{q} W, D_{p}^{q} F, D_{p}^{q} F^{+}, D_{p}^{q} B$ and $D_{p}^{q} B^{+}$ of $X$ FK-spaces are monotone, i.e., if $X \subset Y$, then $E(X) \subset E(Y)$.

Proof. The inclusion map $i: X \rightarrow Y$ is continuous by [11, (4.2.4)], so

$$
\frac{1}{q(n)-p(n)} \sum_{k=p(n)+1}^{q(n)} x^{(k)} \rightarrow x \quad \text { in } X
$$

implies the same in $Y$. This proves the assertion for $D_{p}^{q} S$. For $D_{p}^{q} W$, it follows from the fact that $i$ is weakly continuous by [11, (4.0.11)]. Now, $z \in D_{p}^{q} F^{+}, D_{p}^{q} B^{+}$ if and only if $\left(z_{n} f\left(\delta^{n}\right)\right) \in \sigma_{p}^{q}[s], \sigma_{p}^{q}[b]$, respectively, for all $f \in X^{\prime}$, hence for all $g \in Y^{\prime}$ since $g \mid X \in X^{\prime}$ by [11]. The result follows for $D_{p}^{q} F^{+}, D_{p}^{q} B^{+}$and, thus, for $D_{p}^{q} F, D_{p}^{q} B$.

Since $\left[\sigma_{0}\right]_{p}^{q}$ is an AK-space, we immediately get the following: 
Theorem 2.4. Let $X$ be an FK-space $\supset\left[\sigma_{0}\right]_{p}^{q}$. Then, $\left[\sigma_{0}\right]_{p}^{q} \subset D_{p}^{q} S \subset D_{p}^{q} W$.

Theorem 2.5. Let $X$ be an FK-space $\supset \phi$. Then, $D_{p}^{q} B^{+}=X^{f d[b]}$.

Proof. By Definition 2.1, $z \in D_{p}^{q} B^{+}$if and only if $z \cdot u \in \sigma_{p}^{q}[b]$ for each $u \in X^{f}$. This is precisely the assertion.

Theorem 2.6. Let $X$ be an FK-space $\supset \phi$. Then, $D_{p}^{q} B^{+}$is the same for all $F K$-spaces $Y$ between $\bar{\phi}$ and $X$; i.e., $\bar{\phi} \subset Y \subset X$ implies $D_{p}^{q} B^{+}(Y)=D_{p}^{q} B^{+}(X)$. Here, the closure of $\phi$ is calculated in $X$.

Proof. By Theorem 2.3, we have $D_{p}^{q} B^{+}(\bar{\phi}) \subset D_{p}^{q} B^{+}(Y) \subset D_{p}^{q} B^{+}(X)$. By Theorem 2.5 and $[11,(7.2 .4)]$, the first and the last are equal.

Theorem 2.7. Let $X$ be an FK-space such that $D_{p}^{q} B \supset \bar{\phi}$. Then, $\bar{\phi}$ has $\sigma_{p}^{q}[K]$ and $D_{p}^{q} S=D_{p}^{q} W=\bar{\phi}$.

Proof. Suppose first that $X$ has $\sigma_{p}^{q}[B]$. Define $f_{n}: X \rightarrow X$ by

$$
f_{n}(x)=x-\frac{1}{q(n)-p(n)} \sum_{k=p(n)+1}^{q(n)} x^{(k)} .
$$

Then, $\left\{f_{n}\right\}$ is pointwise bounded, hence equicontinuous by $[11,(7.0 .2)]$. Since $f_{n} \rightarrow$ 0 on $\phi$, then also $f_{n} \rightarrow 0$ on $\bar{\phi}$ by $[11,(7.0 .3)]$. This is the desired conclusion.

Theorem 2.8. Let $X$ be an FK-space $\supset \phi$. Then, $D_{p}^{q} F^{+}=X^{f d}$.

Proof. This may be proved as in Theorem 2.5, with $d$ instead of $d[b]$.

Theorem 2.9. Let $X$ be an $F K$-space $\supset \phi$. Then, $D_{p}^{q} F^{+}$is the same for all $F K$-spaces $Y$ between $\bar{\phi}$ and $X$; i.e., $\bar{\phi} \subset Y \subset X$ implies $D_{p}^{q} F^{+}(Y)=D_{p}^{q} F^{+}(X)$ (the closure of $\phi$ is calculated in $X$ ).

The proof is similar to that of Theorem 2.6.

Lemma 2.10. Let $X$ be an FK-space in which $\bar{\phi}$ has $\sigma_{p}^{q}[K]$. Then, $D_{p}^{q} F^{+}=$ $(\bar{\phi})^{d d}$.

Proof. Observe that $D_{p}^{q} F^{+}=X^{f d}$ by Theorem 2.8. Since $X^{f}=(\bar{\phi})^{f}$ by [11, Thm. 7.2.4], we have $X^{f d}=(\bar{\phi})^{f d}$. Hence, by [8, Thm. 1.9], the result follows.

An FK-space $X$ is said to have $F \sigma_{p}^{q}[K]$ (functional $\sigma_{p}^{q}[K]$ ) if $X \subset D_{p}^{q} F^{+}$, i.e., $X=D_{p}^{q} F$.

Theorem 2.11. Let $X$ be an $F K$-space $\supset \phi$. Then, $X$ has $F \sigma_{p}^{q}[K]$ if and only if $\bar{\phi}$ has $\sigma_{p}^{q}[K]$ and $X \subset(\bar{\phi})^{d d}$.

Proof. Necessity. $X$ has $\sigma_{p}^{q}[B]$ since $D_{p}^{q} F \subset D_{p}^{q} B$, so $\bar{\phi}$ has $\sigma_{p}^{q}[K]$ by Theorem 2.7. The remainder of the proof follows from Lemma 2.10. Sufficiency is given by Lemma 2.10 .

Theorem 2.12. Let $X$ be an FK-space $\supset \phi$. The following are equivalent: 
(i) $X$ has $F \sigma_{p}^{q}[K]$,

(ii) $X \subset\left(D_{p}^{q} S\right)^{d d}$,

(iii) $X \subset\left(D_{p}^{q} W\right)^{d d}$,

(iv) $X \subset\left(D_{p}^{q} F\right)^{d d}$,

(v) $X^{d}=\left(D_{p}^{q} S\right)^{d}$,

(vi) $X^{d}=\left(D_{p}^{q} F\right)^{d}$.

Proof. Observe that (ii) implies (iii) and (iii) implies (iv) and that they are trivial since

$$
D_{p}^{q} S \subset D_{p}^{q} W \subset D_{p}^{q} F
$$

If (iv) is true, then $X^{f} \subset\left(D_{p}^{q} F\right)^{d}=\left(X^{f}\right)^{d d} \subset X^{d}$, so (i) is true by [8, Thm. 1.9]. If (i) holds, then Theorem 2.11 implies that $\bar{\phi}=D_{p}^{q} S$ and that (ii) holds. The equivalence of (v), (vi) with the others is clear.

Theorem 2.13. Let $X$ be an FK-space $\supset \phi$. The following are equivalent:

(i) $X$ has $S \sigma_{p}^{q}[K]$,

(ii) $X$ has $\sigma_{p}^{q}[K]$,

(iii) $X^{d}=X^{\prime}$.

Proof. Clearly, (ii) implies (i). Conversely, if $X$ has $S \sigma_{p}^{q}[K]$, it must have AD for $D_{p}^{q} W \subset \bar{\phi}$ by Theorem 2.2. It also has $\sigma_{p}^{q}[B]$ since $D_{p}^{q} W \subset D_{p}^{q} B$. Thus, $X$ has $\sigma_{p}^{q}[K]$ by Theorem 2.7, which proves that (i) and (ii) are equivalent. Assume that (iii) holds. Let $f \in X^{\prime}$, then there exists $u \in X^{d}$ such that

$$
f(x)=\lim _{n \rightarrow \infty} \frac{1}{q(n)-p(n)} \sum_{k=p(n)+1}^{q(n)} \sum_{j=1}^{k} u_{j} x_{j}
$$

for $x \in X$. Since $f\left(\delta^{j}\right)=u_{j}$, it follows that each $x \in D_{p}^{q} W$, which shows that (iii) implies (i). That (ii) implies (iii) is known (see [6, p. 97]).

Theorem 2.14. Let $X$ be an FK-space $\supset \phi$. The following are equivalent:

(i) $D_{p}^{q} W$ is closed in $X$,

(ii) $\bar{\phi} \subset D_{p}^{q} B$,

(iii) $\bar{\phi} \subset D_{p}^{q} F$,

(iv) $\bar{\phi}=D_{p}^{q} W$

(v) $\bar{\phi}=D_{p}^{q} S$,

(vi) $D_{p}^{q} S$ is closed in $X$.

Proof. (ii) implies (v): By Theorem 2.7, $\bar{\phi}$ has $\sigma_{p}^{q}[K]$, i.e., $\bar{\phi} \subset D_{p}^{q} S$. The opposite inclusion is clear from Theorem 2.2. Note that (v) implies (iv), (iv) implies (iii) and (iii) implies (ii) because

$$
D_{p}^{q} S \subset D_{p}^{q} W \subset \bar{\phi}, D_{p}^{q} W \subset D_{p}^{q} F \subset D_{p}^{q} B ;
$$

(i) implies (iv) and (vi) implies (v) since $\phi \subset D_{p}^{q} S \subset D_{p}^{q} W \subset \bar{\phi}$. Finally, (iv) implies (i) and (v) implies (vi). 


\section{Combinations of neW type subspaces of an FK-SPaCE}

Let $A=\left(a_{n k}\right), n, k=1,2, \ldots$, be an infinite matrix with complex entries and $c_{A}=\{x: A x \in c\}$. Then, $c_{A}$ is an FK-space with seminorms

$$
\rho_{0}(x)=\sup _{n}\left|\sum_{k=1}^{\infty} a_{n k} x_{k}\right| \quad(n=1,2, \ldots)
$$

$\rho_{n}(x)=\left|x_{n}\right|,(n=1,2, \ldots) ;$ and $h_{n}(x)=\sup _{m}\left|\sum_{k=1}^{m} a_{n k} x_{k}\right|(n=1,2, \ldots)$. Also, every $f \in c_{A}^{\prime}$ if and only if

$$
f(x)=\sum_{k=1}^{\infty} \beta_{k} x_{k}+\sum_{n=1}^{\infty} t_{n} \sum_{k=1}^{\infty} a_{n k} x_{k}+\mu \lim _{A} x,
$$

where $t \in l, \mu \in \mathbf{C},\left(\beta_{k}\right) \in c_{A}^{\beta}$, the $\beta$-dual of $c_{A}$ (see $[11,4.4 .3]$ ). The representation is not unique; we say that $A$ is $\mu$-unique if all representations for some $f$ have the same $\mu$. If $A$ is $\mu$-unique, $c_{A} \subset c_{D}, D$ is conull with respect to $A$ if and only if $\mu_{A}\left(\lim _{D}\right)=0$ in $[12]$.

Let $X$ and $Y$ be FK-spaces, $X$ with paranorm $\rho$ and $Y$ with paranorm $s$. It is shown that $Z=X+Y$ with the unrestricted inductive limit topology is an FK-space as in [11, Thm. 4.5.1]. The paranorm $\tau$ of $Z$ is given by

$$
\tau(z)=\inf _{\substack{x+y=z \\ x \in X, y \in Y}}(\rho(x)+s(y)) .
$$

Let $\left\{X^{n}\right\}_{n=1}^{\infty}$ be a sequence of FK-spaces, $\rho_{n}$ be the paranorm of $X^{n}$ and $\left\{s_{n k}\right\}_{k=1}^{\infty}$ be the seminorms of $X^{n}$. Let $Y=\bigcap_{n} X^{n}$. It is well known that $Y$ is an FK-space with paranorm $s=\sum_{n=1}^{\infty} \frac{\rho_{n}}{2^{n}\left(1+\rho_{n}\right)}$ and seminorms $\left\{s_{n k}\right\}_{n, k=1}^{\infty}$.

We now investigate some important subspaces of a locally convex FK-space $X$ containing $\phi$ which are analogous to those given in [5]. To prove the theorems of this section, we use the same technique by DeVos [5].

Theorem 3.1. Let $X, Y$ be $F K$-spaces and $Z=X+Y$. Then, $E(X)+E(Y) \subseteq$ $E(Z)$ for $E=D_{p}^{q} S, D_{p}^{q} W, D_{p}^{q} F$ or $D_{p}^{q} B$.

Proof. Let $E=D_{p}^{q} S$. We take $x \in D_{p}^{q} S(X)$ and $y \in D_{p}^{q} S(Y)$. Then,

$$
\rho\left(\frac{1}{q(n)-p(n)} \sum_{k=p(n)+1}^{q(n)} x^{(k)}-x\right) \rightarrow 0
$$

and

$$
s\left(\frac{1}{q(n)-p(n)} \sum_{k=p(n)+1}^{q(n)} y^{(k)}-y\right) \rightarrow 0
$$

as $n \rightarrow \infty$. Hence,

$$
r\left(\frac{1}{q(n)-p(n)} \sum_{k=p(n)+1}^{q(n)}(x+y)^{(k)}-(x+y)\right)
$$




$$
\leq \rho\left(\frac{1}{q(n)-p(n)} \sum_{k=p(n)+1}^{q(n)} x^{(k)}-x\right)+s\left(\frac{1}{q(n)-p(n)} \sum_{k=p(n)+1}^{q(n)} y^{(k)}-y\right),
$$

which implies that $x+y \in D_{p}^{q} S(Z)$.

Let $E=D_{p}^{q} W$. We take $x \in D_{p}^{q} W(X), y \in D_{p}^{q} W(Y)$ and $f \in Z^{\prime}$. Then, $f \mid X \in X^{\prime}$ and $f \mid Y \in Y^{\prime}$.

$$
\begin{aligned}
& f(x+y)=f(x)+f(y) \\
= & \lim _{n} \frac{1}{q(n)-p(n)} \sum_{k=p(n)+1}^{q(n)} \sum_{j=1}^{k} f\left(\delta^{j}\right) x_{j}+\lim _{n} \frac{1}{q(n)-p(n)} \sum_{k=p(n)+1}^{q(n)} \sum_{j=1}^{k} f\left(\delta^{j}\right) y_{j} \\
= & \lim _{n} \frac{1}{q(n)-p(n)} \sum_{k=p(n)+1}^{q(n)} \sum_{j=1}^{k} f\left(\delta^{j}\right)\left(x_{j}+y_{j}\right) .
\end{aligned}
$$

The proofs for $E=D_{p}^{q} F$ or $D_{p}^{q} B$ are similar, so the details are omitted.

Theorem 3.2. Let $\left\{X^{n}\right\}_{n=1}^{\infty}$ be a sequence of FK-spaces and $Y=\bigcap_{n} X^{n}$. Then, $E(Y)=\bigcap_{n} E\left(X^{n}\right)$ for $E=D_{p}^{q} S, D_{p}^{q} W, D_{p}^{q} F$ or $D_{p}^{q} B$.

Proof. By Theorem 2.3, for each $n, E(Y) \subseteq E\left(X^{n}\right)$, hence $E(Y) \subseteq \bigcap_{n} E\left(X^{n}\right)$ for $E=D_{p}^{q} S, D_{p}^{q} W, D_{p}^{q} F$ or $D_{p}^{q} B$.

Let $z \in \bigcap_{n} D_{p}^{q} S\left(X^{n}\right)$. Then,

$$
s_{n k}\left(\frac{1}{q(r)-p(r)} \sum_{n=p(r)+1}^{q(r)} z^{(n)}-z\right) \rightarrow 0, \quad r \rightarrow \infty,
$$

for each fixed $n$ and $k$, but these are the seminorms for $Y$. Hence,

$$
\lim _{r \rightarrow \infty} \frac{1}{q(r)-p(r)} \sum_{n=p(r)+1}^{q(r)} z^{(n)}=z \quad \text { in } Y,
$$

which implies that $z \in D_{p}^{q} S(Y)$.

Let $z \in \bigcap_{n} D_{p}^{q} W\left(X^{n}\right)$ and $f \in Y^{\prime}$. Then, we have $f=\sum_{j=1}^{h} f_{j}$, where $f_{j} \in$ $\left(X^{j}\right)^{\prime}$ (see [12]). Since

$$
f_{j}\left(\frac{1}{q(r)-p(r)} \sum_{n=p(r)+1}^{q(r)} z^{(n)}\right) \rightarrow f_{j}(z)
$$

for $j=1,2, \ldots, h$. Therefore,

$$
f\left(\frac{1}{q(r)-p(r)} \sum_{n=p(r)+1}^{q(r)} z^{(n)}\right) \rightarrow f(z) .
$$

Hence, $z \in D_{p}^{q} W(Y)$.

The proof for $E=D_{p}^{q} F$ is similar to the one of the previous paragraph, so we omit the details. 
Let $z \in \bigcap_{n} D_{p}^{q} B\left(X^{n}\right)$. Then, for any fixed $l$ and $k$,

$$
s_{l k}\left(\frac{1}{q(r)-p(r)} \sum_{n=p(r)+1}^{q(r)} z^{(n)}\right) \leq H_{l k}
$$

for all $r$. Hence, $z \in D_{p}^{q} B(Y)$.

In [10], let $X$ be an FK-space containing $\phi_{1}$ and

$$
\begin{aligned}
& \zeta^{n}:=e-\frac{1}{q(n)-p(n)} \sum_{k=p(n)+1}^{q(n)} e^{(k)} \\
&=\left(0,0, \ldots, 0, \frac{1}{q(n)-p(n)}, \frac{2}{q(n)-p(n)}, \frac{3}{q(n)-p(n)},\right. \\
&\left.\quad \ldots, \frac{q(n)-p(n)-1}{q(n)-p(n)}, 1,1, \ldots\right),
\end{aligned}
$$

where $e^{(k)}:=\sum_{j=1}^{k} \delta^{j}$. If $\zeta^{n} \rightarrow 0$ in $X$, then $X$ is called strongly deferred Cesàro conull. If the convergence holds in the weak topology in (3.1), then $X$ is called deferred Cesàro conull. Hence, $X$ is deferred Cesàro conull iff

$$
f(e)=\lim _{n} \frac{1}{q(n)-p(n)} \sum_{k=p(n)+1}^{q(n)} \sum_{j=1}^{k} f\left(\delta^{j}\right), \quad \forall f \in X^{\prime} .
$$

Now, we define deferred Cesàro conullity of one FK-space with respect to another.

Definition 3.3. Let $X$ be an FK-space with $D_{p}^{q} W(X) \neq D_{p}^{q} B(X)$ and $Y$ be an FK-space, $X \subseteq Y . Y$ is deferred Cesàro conull with respect to $X$ iff $D_{p}^{q} B(X) \subseteq D_{p}^{q} W(Y)$.

Theorem 3.4. Let $X, Y, Z$ be FK-spaces with $X \subseteq Y \subseteq Z$. Then,

(i) If $Y$ is deferred Cesàro conull with respect to $X$, then $Z$ is deferred Cesàro conull with respect to $X$,

(ii) If $Z$ is deferred Cesàro conull with respect to $X$ and $Y$ is closed in $Z$, then $Y$ is deferred Cesàro conull with respect to $X$.

The proof of the Theorem is clear by Definition 3.3 and Theorem 2.3.

Theorem 3.5. Let $\left\{Y^{n}\right\}_{n=1}^{\infty}$ be FK-spaces such that each $Y^{n}$ is deferred Cesàro conull with respect to $X$. Then, $\bigcap_{n} Y^{n}$ is deferred Cesàro conull with respect to $X$.

The proof of the Theorem is obtained by Definition 3.3 and Theorem 3.2.

Let $E\left(c_{A}\right)=E(A)$ for a matrix $A$ and $E=D_{p}^{q} W$ or $D_{p}^{q} B$ and $\mu_{A}\left(\lim _{D}\right)=$ $\mu_{A}(D)$. For many cases, the following theorem gives an equivalence between Wilansky's and our extensions of deferred Cesàro conullty.

Theorem 3.6. Let $A$ and $D$ be matrices with $D_{p}^{q} W(A) \neq D_{p}^{q} B(A)$ and $c_{A} \subset$ $c_{D} \cdot \mu_{A}(D)=0$ if and only if $c_{D}$ is deferred Cesàro conull with respect to $c_{A}$. 
Proof. Let $c_{D}$ be deferred Cesàro conull with respect to $c_{A}$. For $x \in D_{p}^{q} B(A)$, $\lim _{D} x=\mu_{A}(D) \lim _{A} x+\beta x$.

Now, let $z \in D_{p}^{q} B(A) \backslash D_{p}^{q} W(A)$. Firstly, by [10],

$$
\lim _{A}\left(z-\frac{1}{q(n)-p(n)} \sum_{k=p(n)+1}^{q(n)} z^{(k)}\right)
$$

is not convergent to zero. Also, since $D_{p}^{q} B(A) \subset c_{A}$ and $\gamma \in c_{A}^{\beta}$, we obtained

$$
\begin{aligned}
\gamma\left(z-\frac{1}{q(n)-p(n)} \sum_{k=p(n)+1}^{q(n)} z^{(k)}\right) & =\gamma\left(z-\frac{1}{q(n)-p(n)} \sum_{k=p(n)+1}^{q(n)} \sum_{j=1}^{k} z_{j} \delta^{j}\right) \\
& =\frac{1}{q(n)-p(n)} \sum_{k=p(n)+1}^{q(n)} \sum_{j=k+1}^{\infty} z_{j} \delta^{j} .
\end{aligned}
$$

By hypothesis, for each $f \in\left(c_{D}\right)^{\prime}$,

$$
f\left(z-\frac{1}{q(n)-p(n)} \sum_{k=p(n)+1}^{q(n)} z^{(k)}\right) \rightarrow 0 .
$$

In particular, we take $f=\lim _{D} \in\left(c_{D}\right)^{\prime}$. Thus,

$$
\lim _{D}\left(z-\frac{1}{q(n)-p(n)} \sum_{k=p(n)+1}^{q(n)} z^{(k)}\right) \rightarrow 0 .
$$

Conversely, let $f \in\left(c_{D}\right)^{\prime}$. By [12], we have $\mu_{A}(f)=\mu_{D}(f) \cdot \mu_{A}(D)=0$. Hence, $f(x)=t(A x)+\beta x$ for $x \in c_{A}$.

Now, we are able to write

$$
f(x)=\mu_{A}(f) \lim _{A} x+\beta x
$$

with $\gamma=t A+\beta$ for $x \in D_{p}^{q} B$ by [11]. Therefore, we get $f(x)=\gamma x$ for $x \in D_{p}^{q} B(A)$, which implies that $x \in D_{p}^{q} W(D)$. So, $c_{D}$ is deferred Cesàro conull with respect to $c_{A}$.

We establish some relations among the subspaces $D_{p}^{q} S, D_{p}^{q} W, D_{p}^{q} F, D_{p}^{q} F^{+}$.

Remark 3.7. Let $X$ be an FK-space such that weakly convergent sequences are convergent in the FK-topology, $A$ be a matrix such that $X_{A} \supset \phi$. The subspaces $D_{p}^{q} S, D_{p}^{q} W$ and $D_{p}^{q} F$ are calculated in $X_{A}$.

Lemma 3.8. If $X$ is as in Remark 3.7, then, for $X$ itself, we have $D_{p}^{q} S=$ $D_{p}^{q} W=D_{p}^{q} F=D_{p}^{q} F^{+}$.

Proof. The inclusions $D_{p}^{q} S \subset D_{p}^{q} W \subset D_{p}^{q} F \subset D_{p}^{q} F^{+}$are trivial by definitions. Conversely, if $x \in D_{p}^{q} F^{+}$, then

$$
\left\{\frac{1}{q(n)-p(n)} \sum_{k=p(n)+1}^{q(n)} x^{(k)}\right\}
$$


is weakly Cauchy, hence by $[11,12.0 .1]$ is Cauchy in the FK-topology of $X$, so convergent, say

$$
\left\{\frac{1}{q(n)-p(n)} \sum_{k=p(n)+1}^{q(n)} x^{(k)}\right\} \rightarrow y .
$$

Since $x^{(k)} \rightarrow x$ in $w$, we have

$$
\left\{\frac{1}{q(n)-p(n)} \sum_{k=p(n)+1}^{q(n)} x^{(k)}\right\} \rightarrow x \quad \text { in } w .
$$

By the contiunity of $i: X \rightarrow w, y=x$, and $x \in D_{p}^{q} S$.

Now, we note that if $X$ is an FK-space containing $\phi_{1}$, then

$$
D_{p}^{q} F^{+}=X^{f d}
$$

To see this, it is enough to take $\sigma_{p}^{q}[s]$ instead of $c s$ in [11, Thm. 10.4.2]. If $X$ is also $\sigma_{p}^{q}[K]$, we can conclude that $X^{d d}=X$ by $(3.2)$. We have $X^{d d}=D_{p}^{q} F=$ $D_{p}^{q} F^{+} \subset X$, hence the result follows.

Theorem 3.9. With $X, A$ as in Remark 3.7, for the $X_{A}$, we have $D_{p}^{q} S=$ $D_{p}^{q} W=D_{p}^{q} F=D_{p}^{q} F^{+}$.

Proof. If $x \in D_{p}^{q} F^{+}$, then

$$
\left\{\frac{1}{q(n)-p(n)} \sum_{k=p(n)+1}^{q(n)} x^{(k)}\right\}
$$

is weakly Cauchy, hence it is Cauchy in the FK-topology of $X$, so convergent. Since by Lemma 12.0.2 of [11] the matrix mapping $A: X_{A} \rightarrow X$ is continuous,

$$
\left\{\frac{1}{q(n)-p(n)} \sum_{k=p(n)+1}^{q(n)} A x^{(k)}\right\}
$$

is convergent in $X$, say

$$
\left\{\frac{1}{q(n)-p(n)} \sum_{k=p(n)+1}^{q(n)} A x^{(k)}\right\} \rightarrow y .
$$

On the other hand, by $[11,4.3 .8],\left(w_{A}, \rho \cup h\right)$ is an AK-space. Hence, it is also a $\sigma_{p}^{q}[K]$-space. Hence,

$$
\left\{\frac{1}{q(n)-p(n)} \sum_{k=p(n)+1}^{q(n)} x^{(k)}\right\} \rightarrow x .
$$

The matrix mapping $A: w_{A} \rightarrow w$ is continuous, and therefore

$$
\left\{\frac{1}{q(n)-p(n)} \sum_{k=p(n)+1}^{q(n)} A x^{(k)}\right\} \rightarrow A x \quad \text { in } w .
$$


Since $X \subset w$ and $X$ is complete, $A x=y$. We have

$$
\left\{\frac{1}{q(n)-p(n)} \sum_{k=p(n)+1}^{q(n)} A x^{(k)}\right\} \rightarrow A x \quad \text { in } X .
$$

That is,

$$
\begin{aligned}
r\left(A x-\frac{1}{q(n)-p(n)} \sum_{k=p(n)+1}^{q(n)} A x^{(k)}\right) & \\
& =(r \circ A)\left(x-\frac{1}{q(n)-p(n)} \sum_{k=p(n)+1}^{q(n)} x^{(k)}\right) \rightarrow 0
\end{aligned}
$$

as $n \rightarrow \infty$, where $r$ is a typical seminorm of $X$. Hence, $x \in D_{p}^{q} S$, which proves Theorem 3.9.

\subsection{Replaceability, deferred Cesàro conullity of $l_{A}$}

Recall that a matrix $A$ with $l_{A} \supset \phi$ is called $l$-replaceable if there is a matrix $D=\left(d_{n k}\right)$ with $l_{D}=l_{A}$, and $\sum_{n} d_{n k}=1, k \in \mathbb{N}$ (see [9]). It is easy to see that $A$ is replaceable if and only if there exists $f \in l_{A}^{\prime}$ with $f\left(\delta^{k}\right)=1(k \in \mathbb{N})$, namely, $f=\sum_{D}$.

Theorem 3.10. Suppose that $D_{p}^{q} F=l_{A}$. Then, $A$ is l-replaceable if and only if $l_{A} \subset \sigma_{p}^{q}[s]$.

Proof. Assume that $A$ is $l$-replaceable. Then, it follows from [9] that $A$ is $l$ replaceable if and only if there is $f \in l_{A}^{\prime}$ such that $f\left(\delta^{j}\right)=1$ for all $j \in \mathbb{N}$. Since $D_{p}^{q} F=l_{A}$,

$$
\lim _{n} \frac{1}{q(n)-p(n)} \sum_{k=p(n)+1}^{q(n)} \sum_{j=1}^{k} x_{j} f\left(\delta^{j}\right)=\lim _{n} \frac{1}{q(n)-p(n)} \sum_{k=p(n)+1}^{q(n)} \sum_{j=1}^{k} x_{j}
$$

exists for all $x \in l_{A}$, hence $x \in \sigma_{p}^{q}[s]$.

Conversely, if $l_{A} \subset \sigma_{p}^{q}[s]$, then

$$
f(x)=\frac{1}{q(n)-p(n)} \sum_{k=p(n)+1}^{q(n)} \sum_{j=1}^{k} x_{j}
$$

defines an element $f$ of $l_{A}^{\prime}$, and we get $f\left(\delta^{\nu}\right)=1(\nu=1,2, \ldots)$.

We now establish a relation between deferred Cesàro conullity and replaceability.

Theorem 3.11. If the space $l_{A}$ is deferred Cesàro conull, then $A$ is not l-replaceable.

Proof. Suppose that $A$ is $l$-replaceable. Then, it follows from [9] that $A$ is $l$ replaceable if and only if there is $f \in l_{A}^{\prime}$ such that $f\left(\delta^{j}\right)=1$ for all $j \in \mathbb{N}$. Hence, 
we have

$$
\begin{aligned}
& f(e)-\frac{1}{q(n)-p(n)} \sum_{k=p(n)+1}^{q(n)} \sum_{j=1}^{k} f\left(\delta^{j}\right) \\
& =\left(f(e)-\frac{1}{q(n)-p(n)} \sum_{k=p(n)+1}^{q(n)} \sum_{j=1}^{k}(1)\right)=\left(f(e)-\frac{1}{q(n)-p(n)} \sum_{k=p(n)+1}^{q(n)}(k)\right) \\
& =\left(f(e)-\frac{1}{q(n)-p(n)} \frac{(q(n)-p(n))(q(n)+p(n)+1)}{2}\right) \\
& =\left(f(e)-\frac{q(n)+p(n)+1}{2}\right)
\end{aligned}
$$

is not convergent as $n \rightarrow \infty$. So, $l_{A}$ is not deferred Cesàro conull.

\section{Conclusion}

Deferred Cesàro conullity is a generalization of the Cesàro conullity and $C_{\lambda}$ conullity. Indeed, if $p(n)=0$ and $q(n)=n$, we get Cesàro conullity and if $p(n)=0$ and $q(n)=\lambda(n)$, where $\{\lambda(n)\}$ is a strictly increasing sequence of positive integers, we get $C_{\lambda}$ conullity.

\section{REFERENCES}

[1] R. P. Agnew, On deferred Cesàro means, Annals of Math. 33 (1932), 413-421.

[2] J. Boos, Classical and Modern Methods in Summability, Oxford University Press, New York, 2000.

[3] M. Buntinas, Convergent and bounded Cesàro sections in FK-spaces, Math. Z. 121 (1971), 191-200.

[4] I. Dağadur, On some subspaces of an FK-space, Math. Commun. 7 (2002), 15-20.

[5] R. DeVos, Combinations of distinguished subsets and conullity, Math. Z. 192 (1986), 447451.

[6] G. Goes and S. Goes, Sequences of bounded variation and sequences of Fourier coefficients I, Math. Z. 118 (1970), 93-102.

[7] G. Goes, Sequences of bounded variation and sequences of Fourier coefficients II, J. Math. Anal. Appl. 39 (1972), 477-494.

[8] G. Goes, Summan von FK-Räumen funktionale Abschnıttskonvergenz und Umkehrsatz, Tôhoku. Math. Journ. 26 (1974), 487-504.

[9] M. S. Macphail and C. Orhan, Some properties of absolute summability domains, Analysis 9 (1989), 317-322.

[10] Ş. Sezgek and İ. Dağadur, Deferred Cesàro conull FK spaces, submitted.

[11] A. Wilansky, Summability through Functional Analysis, North Holland, 1984.

[12] A. Wilansky, The $\mu$ property of FK-spaces, Comment. Math. 21 (1978), 371-380.

[13] K. Zeller, Allgemeine Eigenschaften von limitierungsverfahren, Math. Z. 53 (1951), 463487.

Şeyda Sezgek, Department of Mathematics, Mersin University, 33343, Mersin, Turkey e-mail: seydasezgek@gmail.com

İlhan Dağadur, Department of Mathematics, Mersin University, 33343, Mersin, Turkey e-mail: ilhandagadur@yahoo.com 